\title{
Perfil dos atendimentos das unidades de suporte avançado do SAMU regional metropolitano do Paraná
}

\author{
Profile of the medical care provided by the emergency medical service SAMU metropolitan \\ regional of Paraná
}
Perfil de atención de las unidades de soporte avanzado del SAMU regional metropolitano de Paraná

Isabeli Emily Chevonik ${ }^{1 *}$, Larissa Marcondes ${ }^{1}$, Junio César da Silva ${ }^{1}$, Thais Lazaroto Roberto Cordeiro ${ }^{1}$, Gerson Martins Albuquerque ${ }^{1}$.

\section{RESUMO}

Objetivo: Descrever o perfil dos atendimentos realizados pelas Unidades de Suporte Avançado (USA) do Serviço de Atendimento Móvel de Urgência (SAMU) Regional Metropolitano do Paraná. Métodos: Estudo retrospectivo, descritivo e quantitativo. A amostra estratificada foi de 388 fichas de atendimentos das USAs do SAMU Regional Metropolitano do Paraná, referente ao período de $1^{\circ}$ de agosto de 2018 a 31 de julho de 2019. A coleta se deu nos meses de abril a agosto de 2020. A análise foi realizada através do Teste QuiQuadrado, pelo software CORE R 4.0.3. Resultados: Observou-se maior predominância do sexo masculino com $271(58 \%)$, com mais de 60 anos ( $n=182 ; 48,7 \%)$, atendimentos clínicos ( $n=138 ; 71,5 \%)$, sendo a dor torácica $(n=79 ; 21,5 \%)$, Acidente Vascular Cerebral $(n=32 ; 8,7 \%)$ e óbito $(n=30 ; 8,2 \%)$ os três de maior prevalência. Conclusão: As USAs do SAMU Regional Metropolitano-PR realizaram predominantemente atendimentos clínicos, e esses foram diretamente proporcionais ao aumento da idade. Quanto ao objetivo do chamado, a maioria foi transferências. Levando em consideração os principais diagnósticos atendidos, é notável que as USAs cumprem seu papel de assistência ao paciente de maior gravidade.

Palavras-chaves: Serviços médicos de emergência, Perfil de saúde, Unidades móveis de saúde.

\begin{abstract}
Objective: To describe the profile of the services provided by the Advanced Support Units (USA) of the Mobile Emergency Service (SAMU) Metropolitan Regional of Paraná. Methods: Retrospective, descriptive and quantitative study. The stratified sample consisted of 388 care records from the USAs of the SAMU Metropolitan Regional of Paraná, referring to the period from August 1, 2018 to July 31, 2019. The data study took place from April to August 2020. The analysis was performed through the Chi-Square Test, using the CORE R 4.0.3 software. Results: There was a greater predominance of males with $271(58 \%)$, over 60 years old $(n=182 ; 48.7 \%)$, clinical care $(n=138 ; 71.5 \%)$, being chest pain $(n=79 ; 21.5 \%)$, Stroke $(n=32 ; 8.7 \%)$ and death $(n=30 ; 8.2 \%)$ the three most prevalent. Conclusion: The USAs of SAMU Metropolitan-PR performed predominantly clinical consultations, and these were directly proportional to the increase in age. As for the purpose of the call, the majority were transfers. Considering the main diagnoses attended, it is notable that the USAs fulfill their role of assisting the most serious patient.
\end{abstract}

Keywords: Emergency medical services, Health profile, Mobile health units.

\section{RESUMEN}

Objetivo: Describir el perfil de atención ofrecido por las Unidades de Soporte Avanzado (USA) del Servicio de Atención Móvil de Urgencia (SAMU) Regional Metropolitano de Paraná. Métodos: Estudio retrospectivo, descriptivo y cuantitativo. El muestreo estratificado estuvo constituido por 388 historias clínicas de las USAs del SAMU Regional Metropolitano de Paraná, del período comprendido entre el 1 de agosto de 2018 y el 31

\footnotetext{
${ }^{1}$ Secretaria Municipal de Saúde de Curitiba (SMS), Curitiba - PR. *E-mail: isabelichevonik@gmail.com
} 
de julio de 2019. Los datos se recogieron de abril a agosto de 2020. El análisis se realizó mediante la prueba de Chi-Cuadrado, por medio del software CORE R 4.0.3. Resultados: Se observó un mayor predominio de varones con $271(58 \%)$, mayores de 60 años $(n=182 ; 48,7 \%)$, asistencias clínicas $(n=138 ; 71,5 \%)$, siendo el dolor torácico ( $n=79 ; 21,5 \%)$, el Accidente cerebrovascular $(n=32 ; 8,7 \%)$ y la muerte $(n=30 ; 8,2 \%)$ los tres con mayor prevalencia. Conclusión: Las USAs del SAMU Regional Metropolitano-PR realizaron una atención predominantemente clínica, y ésta fue directamente proporcional al aumento de la edad. En cuanto al objeto de la llamada, la mayoría eran traslados. Teniendo en cuenta los principales diagnósticos atendidos, cabe destacar que las USAs cumplen su función de asistir al paciente más grave.

Palabras clave: Servicios médicos de urgencia, Perfil de salud, Unidades móviles de salud.

\section{INTRODUÇÃO}

Frequentemente os termos urgência e emergência são utilizados como sinônimos, entretanto possuem significados distintos. Emergência refere-se ao agravo de saúde que oferece risco iminente de morte com necessidade de intervenção médica imediata. Urgência refere-se a situação imprevista de injúria à saúde podendo ou não oferecer risco de morte iminente e necessita de assistência médica (BRASIL, 2014).

Com a transição demográfica, consequentemente o aumento das doenças crônicas, principalmente relacionadas à problemas cardiovasculares, juntamente com o rápido crescimento populacional, violência urbana e acidentes automobilísticos que resultaram no aumento de agravos relacionados às causas externas, influenciaram os grandes índices de procura da população por serviços de emergência (ABREU, et al., 2019; CASTRO RR, et at., 2020).

Diante dessa realidade percebeu-se a necessidade da organização dos serviços de emergência dentro do Sistema Único de Saúde (SUS). Com objetivo de proporcionar acesso universal e equidade na distribuição dos recursos, o Ministério da Saúde em por meio da ํㅡ 2048/2002 estabeleceu os princípios e diretrizes para funcionamento dos serviços de emergência, definiu o atendimento de emergência em componente fixo e móvel (BRASIL, 2002).

Para ser o componente móvel foi criado o Serviço de Atendimento Móvel de Urgência (SAMU) que integra a Rede de Urgência e Emergência (RUE) nacional, configurando-se como uma modalidade de atendimento 24 horas que objetiva chegar precocemente à vítima, prestar atendimento e disponibilizar deslocamento adequado a uma porta de entrada do SUS, possibilitando maior sobrevida e a diminuição dos agravos de saúde. Foi criado e regulamentado pelas Portarias no 2048/2002 e no 1.864/2003, sendo o principal elemento da Política Nacional de Atenção à Urgências (PNAU) (BRASIL, 2002; BRASIL, 2003).

O SAMU é vinculado a uma Central de Regulação específica, para solicitação do serviço é necessário realizar uma ligação telefônica ao número 192. O solicitante é primeiramente atendido por um Técnico Auxiliar de Regulação Médica (TARM), que realiza a coleta dos principais dados da ocorrência e após transfere a ligação para um médico regulador, esse é responsável por avaliar cada caso e decidir a conduta mais apropriada, podendo ser uma orientação médica ou envio de uma equipe de atendimento ao local da ocorrência (BATTISTI GR, et al., 2019).

O SAMU conta com Unidades de Suporte Básico (USBs) e Unidades de Suporte Avançado (USAs), além de aeronaves, embarcações e motolâncias. No Brasil, estão em funcionamento um total de 2.965, destas 567 USA, 2.382 UBS, 217 Motolâncias, 9 Embarcações e 7 Aeronaves. As UBS são responsáveis pelo transporte e atendimento pré-hospitalar de pacientes avaliados e classificados que não necessitam de assistência médica durante o percurso. A equipe é composta por técnico de enfermagem e condutor socorrista. As USA são ambulâncias destinadas à assistência a pacientes críticos de atendimentos Pré-Hospitalar ou remoções, que necessitam de materiais específicos e equipe composta por médico, enfermeiro e condutor socorrista (BRASIL, 2002; FERREIRA AM, 2017).

A cidade de Curitiba, capital do estado do Paraná, dispõe de uma área de $434.892 \mathrm{~km}^{2}$ e uma população de 1.751.907 habitantes. Sua Região Metropolitana possui 29 municípios, composta por uma área de 
$15.418,543 \mathrm{~km}^{2}$ com população de 3.572,326 habitantes. A Portaria n. 1.864/GM estabelece a proporção de uma UBS para cada 100.000 a 150.000 habitantes e uma USA para cada 400.000 a 450.000 habitantes (REZENDE DA, et al., 2021; BRASIL, 2003).

$\mathrm{Na}$ busca de literatura científica quanto ao perfil dos atendimentos do SAMU, foram encontrados diversos estudos pelo território brasileiro, porém no Estado do Paraná essa temática ainda é pouco explorada, e esse fato estimulou a realização deste estudo (DORR MR, et al., 2020; TIBÃES HBB, et al., 2018; SOARES MKP, et al., 2018).

Cotidianamente, percebe-se que há um aumento no número de ocorrências gerados para o SAMU, sendo assim, considera-se importante conhecer e analisar os atendimentos realizados por esse serviço, para uma reflexão promotora de mudanças, tornando o serviço cada vez mais eficaz. Diante disso, questionou-se: Qual o perfil dos atendimentos realizados pelo Suporte Avançado do SAMU Regional Metropolitano-PR? Dessa forma, objetiva-se descrever o perfil dos atendimentos realizados pelas Unidades de Suporte Avançado do Serviço de Atendimento Móvel de Urgência Regional Metropolitano do Paraná.

\section{MÉTODOS}

Trata-se de um estudo retrospectivo, descritivo, de abordagem quantitativa. Os dados foram coletados das informações das fichas de atendimento das 14 USAs do banco de dados do SAMU Regional Metropolitano PR.

Os critérios de inclusão foram: fichas de atendimento preenchidas pelos profissionais nas ocorrências realizadas, referentes ao período de $1^{\circ}$ de agosto de 2018 a 31 de julho de 2019 , das USAs do SAMU Regional Metropolitano-PR alocadas em seis municípios. Foram excluídas deste estudo as fichas preenchidas com letra ilegível ou que receberam o cancelamento da ocorrência.

Para o cálculo do tamanho da amostra considerou-se o número de atendimentos mensais das Unidades de Suporte Avançado do SAMU no período de $1^{\circ}$ de agosto de 2018 a 31 de julho de 2019 (30.951 atendimentos). Para esta pesquisa foi utilizada uma amostra estratificada calculada usando o número total de atendimentos, com $95 \%$ de confiança e margem de erro igual a $5 \%$. Dessa forma, chegando à amostra de 388 fichas de atendimentos, que foram escolhidas manualmente nos locais onde são armazenadas, conforme sorteio calculado estatisticamente, e repassadas inteiramente para uma planilha do Excel®.

A coleta se realizou nos meses de abril a agosto de 2020, nas dependências dos locais onde as fichas são armazenadas (Departamento de Urgência e Emergência e bases da região metropolitana). Após a coleta, os dados foram tabulados e categorizados utilizando uma planilha do Excel®.

A análise descritiva foi obtida através dos cálculos de média, mediana, amplitude, frequência simples e absoluta; para os testes de associação empregou-se o Teste Qui-Quadrado, utilizando o software $R$ versão 4.0.3 (R CORE TEAM, 2020).

Para o acesso às fichas, a pesquisa respeitou os preceitos éticos da Resolução no 466/12 do Conselho Nacional de Saúde e foi aprovada pelo Comitê de Ética em Pesquisa da Secretaria Municipal de Saúde de Curitiba - SMS, com o CAAE - 26483119.2.0000.0101, sob o parecer no 3.764.811 (BRASIL, 2012).

\section{RESULTADOS}

Das 388 fichas de atendimento selecionadas como amostra estratificada, houve predominância do sexo masculino ( $n=217 ; 58 \%)$, com faixa etária maior de 60 anos, $(n=183 ; 48,7 \%)$, sendo a média de 54,32 anos e a mediana de 58, conforme demostrado na Tabela 1. Quanto à natureza do chamado, 195 fichas não foram preenchidas e, das preenchidas ( $n=193)$, houve predominância $(n=138 ; 71 \%)$ dos atendimentos clínicos. No que se refere ao objetivo da ocorrência, uma ficha não foi preenchida e $(n=203 ; 52,5 \%)$ foram de transferências; o turno do chamado mais recorrente foi o período diurno, com ( $n=203 ; 55,9 \%$ ), conforme apresentado na Tabela 1. 
Tabela 1 - Distribuição dos atendimentos realizados pelas Unidades de Suporte Avançado do SAMU Regional Metropolitano/PR, segundo o sexo, faixa etária, natureza dos chamados, objetivo dos chamados, turno dos chamados.

\begin{tabular}{|c|c|c|}
\hline Variável & $\mathbf{N}^{*}$ & $\%$ \\
\hline \multicolumn{3}{|l|}{ Sexo $(n=374)$} \\
\hline Masculino & 217 & 58 \\
\hline Feminino & 157 & 42 \\
\hline \multicolumn{3}{|l|}{ Faixa etária $(n=376)$} \\
\hline $0-18$ anos & 21 & 5,6 \\
\hline $18-59$ anos & 172 & 45,7 \\
\hline$>60$ anos & 183 & 48,7 \\
\hline Variável & $\mathrm{N}^{*}$ & $\%$ \\
\hline \multicolumn{3}{|c|}{ Natureza do chamado $(n=192)$} \\
\hline Clínicos & 138 & 71,5 \\
\hline Psiquiátricos & 19 & 9,8 \\
\hline Trauma & 10 & 5,2 \\
\hline Pediátricos & 19 & 9,8 \\
\hline Ginecológica/obstétrica & 6 & 3,1 \\
\hline \multicolumn{3}{|c|}{ Objetivo do chamado $(n=387)$} \\
\hline Atendimento Primário & 184 & 47,5 \\
\hline Transferência & 203 & 52,5 \\
\hline \multicolumn{3}{|l|}{ Turno $(n=363)$} \\
\hline Diurno & 203 & 55,9 \\
\hline Noturno & 160 & 44,1 \\
\hline
\end{tabular}

Nota: * Foram consideradas somente as fichas preenchidas, por esse motivo "n" variável.

Fonte: Chevonik IE, et al., 2021.

Quanto aos antecedentes, destaca-se que 239 fichas não estavam preenchidas. Das que continham informação ( $n=149)$, ( $n=80 ; 53,7 \%)$ possuíam Hipertensão Arterial Sistêmica (HAS); $(n=60 ; 40,3 \%)$, Diabetes Mellitus (DM); e ( $n=28 ; 18,8 \%)$, doença cardíaca prévia. Destaca-se que um mesmo paciente pode apresentar mais de uma comorbidade (Tabela 2).

Tabela 2 - Antecedentes dos pacientes atendidos pelas Unidades de Suporte Avançado do SAMU Regional Metropolitano/PR. $\mathrm{n}=255$.

\begin{tabular}{lccc}
\hline Antecedentes & $\mathbf{N}$ & $\%^{*}$ & \% Multi ${ }^{* *}$ \\
\hline Hipertensão & 80 & 31,4 & 53,7 \\
Diabetes & 60 & 23,5 & 40,3 \\
Doença Cardíaca & 28 & 11,0 & 18,8 \\
AVC & 13 & 5,1 & 8,7 \\
Alcoolismo & 11 & 4,3 & 7,4 \\
Convulsões & 9 & 3,5 & 6,0 \\
Abuso de drogas & 9 & 3,5 & 6,0 \\
Doença Renal & 7 & 2,7 & 4,7 \\
Doença Respiratória & 7 & 2,7 & 4,7 \\
DPOC & 1 & 0,4 & 0,7 \\
Outros & 30 & 11,8 & 20,1 \\
\hline Total & 255 & 100,0 & 171,1 \\
\hline
\end{tabular}

Nota: * \% em relação ao total de respondentes (149); \% em relação ao total de respostas múltiplas (255). Legenda: AVC: acidente vascular cerebral; DPOC: doença pulmonar obstrutiva crônica.

Fonte: Chevonik IE, et al., 2021. 
Entre os principais diagnósticos encontrados, apresentados na Tabela 2, dos sete mais prevalentes, destacam-se dor torácica com $(n=79 ; 21,5 \%)$, seguido de Acidente Vascular Cerebral (AVC) com $(n=32$; $8,7 \%)$, com maior ocorrência no sexo masculino para ambos, e óbito com ( $n=30 ; 8,2 \%)$, com maior ocorrência no sexo feminino, no período diurno (Tabela 3).

Quando associados os principais diagnósticos à faixa etária, o estudo evidencia que os pacientes com mais de 60 anos estão representados por uma frequência maior de dor torácica, AVC e óbito; e, entre a faixa etária de 18 a 59 anos, destacam-se traumatismo e crise convulsiva.

Tabela 3 - Associação dos principais diagnósticos encontrados em atendimentos realizados pelas Unidade de Suporte Avançado do SAMU Regional Metropolitano/PR, com as variáveis sexo, faixa etária e turno do chamado.

\begin{tabular}{|c|c|c|c|c|c|c|c|}
\hline Variáveis & $\begin{array}{c}\text { Dor } \\
\text { Torácica } \\
(\mathbf{n = 7 9 )}\end{array}$ & $\begin{array}{c}\text { AVC } \\
(n=32)\end{array}$ & $\begin{array}{l}\text { Óbito } \\
(n=30)\end{array}$ & $\begin{array}{l}\text { Surto } \\
(n=25)\end{array}$ & $\begin{array}{c}\text { Trauma } \\
(n=23)\end{array}$ & $\begin{array}{c}\text { Hemorragia } \\
\text { Digestiva } \\
(n=17)\end{array}$ & $\begin{array}{c}\text { Crise } \\
\text { Convulsiva } \\
(n=16)\end{array}$ \\
\hline \multicolumn{8}{|l|}{ Sexo } \\
\hline $\begin{array}{l}\text { Não } \\
\text { preenchido }\end{array}$ & 2 & 1 & 0 & 0 & 3 & 0 & 1 \\
\hline Feminino & $\begin{array}{l}40,3 \% \\
(n=31)\end{array}$ & $\begin{array}{l}48,4 \% \\
(n=15)\end{array}$ & $\begin{array}{l}56,7 \% \\
(n=17)\end{array}$ & $\begin{array}{l}40,0 \% \\
(n=10)\end{array}$ & $\begin{array}{c}30,0 \% \\
(n=6)\end{array}$ & $\begin{array}{c}29,4 \% \\
(n=5)\end{array}$ & $\begin{array}{c}53,3 \% \\
(n=8)\end{array}$ \\
\hline Masculino & $\begin{array}{l}59,7 \% \\
(n=46) \\
\end{array}$ & $\begin{array}{l}51,6 \% \\
(n=16) \\
\end{array}$ & $\begin{array}{l}43,3 \% \\
(n=13) \\
\end{array}$ & $\begin{array}{c}60,00 \% \\
(n=15) \\
\end{array}$ & $\begin{array}{c}70,00 \% \\
(n=14) \\
\end{array}$ & $\begin{array}{l}70,6 \% \\
(n=12) \\
\end{array}$ & $\begin{array}{l}51,6 \% \\
(n=16) \\
\end{array}$ \\
\hline \multicolumn{8}{|l|}{ Faixa Etária } \\
\hline $\begin{array}{l}\text { Não } \\
\text { Preenchido }\end{array}$ & 1 & 1 & 1 & 0 & 2 & 0 & 1 \\
\hline $0-18$ anos & $\begin{array}{l}2,6 \% \\
(n=2)\end{array}$ & $\begin{array}{l}0,0 \% \\
(n=0)\end{array}$ & $\begin{array}{l}3,4 \% \\
(n=1)\end{array}$ & $\begin{array}{l}4,0 \% \\
(n=1)\end{array}$ & $\begin{array}{l}0,0 \% \\
(n=0)\end{array}$ & $\begin{array}{l}0,0 \% \\
(n=0)\end{array}$ & $\begin{array}{c}26,7 \% \\
(n=4)\end{array}$ \\
\hline $18-59$ anos & $\begin{array}{c}37,2 \% \\
(n=9)\end{array}$ & $\begin{array}{c}19.4 \% \\
(n=6)\end{array}$ & $\begin{array}{l}34,5 \% \\
(n=10)\end{array}$ & $\begin{array}{l}76,0 \% \\
(n=19)\end{array}$ & $\begin{array}{l}81,0 \% \\
(n=17)\end{array}$ & $\begin{array}{l}47,1 \\
(n=8)\end{array}$ & $\begin{array}{c}60,0 \% \\
(n=9)\end{array}$ \\
\hline$>60$ anos & $\begin{array}{l}60,3 \% \\
(n=47)\end{array}$ & $\begin{array}{l}80,6 \% \\
(n=25)\end{array}$ & $\begin{array}{l}52,4 \% \\
(n=11)\end{array}$ & $\begin{array}{c}20,0 \% \\
(n=5)\end{array}$ & $\begin{array}{c}19,0 \% \\
(n=4)\end{array}$ & $\begin{array}{c}52,9 \% \\
(n=9)\end{array}$ & $\begin{array}{c}52,9 \% \\
(n=2)\end{array}$ \\
\hline \multicolumn{8}{|l|}{$\begin{array}{l}\text { Turno do } \\
\text { Chamado }\end{array}$} \\
\hline $\begin{array}{l}\text { Não } \\
\text { Preenchido }\end{array}$ & 5 & 4 & 5 & 3 & 0 & 1 & 0 \\
\hline Diurno & $\begin{array}{l}60,8 \% \\
(n=45)\end{array}$ & $\begin{array}{l}60,7 \% \\
(n=17)\end{array}$ & $\begin{array}{l}80,0 \% \\
(n=20)\end{array}$ & $\begin{array}{l}50,0 \% \\
(n=11)\end{array}$ & $\begin{array}{l}43,5 \% \\
(n=10)\end{array}$ & $\begin{array}{c}56,2 \% \\
(n=9)\end{array}$ & $\begin{array}{c}50,0 \% \\
(n=8)\end{array}$ \\
\hline Noturno & $\begin{array}{l}39,2 \% \\
(n=29)\end{array}$ & $\begin{array}{l}39,3 \% \\
(n=11)\end{array}$ & $\begin{array}{c}20,0 \% \\
(n=5)\end{array}$ & $\begin{array}{l}50,0 \% \\
(n=11)\end{array}$ & $\begin{array}{l}56,5 \% \\
(n=13)\end{array}$ & $\begin{array}{c}43,8 \% \\
(n=7)\end{array}$ & $\begin{array}{c}50,0 \% \\
(n=8)\end{array}$ \\
\hline
\end{tabular}

Legenda: AVC: acidente vascular cerebral.

Fonte: Chevonik IE, et al., 2021.

\section{DISCUSSÃO}

Em relação às características sociodemográficas, observa-se que a prevalência masculina está presente em todas as regiões do Brasil, corroborando com este estudo. Na Região Sul, no Estado do Rio Grande do Sul, estudo que objetivou analisar os atendimentos na Central de Regulação de Urgência/SAMU, com 280.754 atendimentos, apresentou 56,7\% dos atendimentos do sexo masculino. Na Região Centro-Oeste, em Minas Gerais, uma pesquisa avaliou 117.289 atedimentos do SAMU com o objetivou de descrever o perfil dos atendimentos, 55,22\% eram do sexo masculino. E, na Região Nordeste, no Rio Grande do Norte, a prevalência foi de 64,6\% para o sexo masculino (DORR MR, et al., 2020; TIBÃES HBB, et al., 2018; SOARES MKP, et al., 2018). 
Entretanto, um estudo com o objetivo de avaliar os padrões de demanda dos atendimentos, através da análise das ligações realizadas para o serviço do SAMU em municípios do Rio de Janeiro, onde foi avaliado um volume de 590.902 ligações, mostrou a prevalência de atendimentos a mulheres de meia-idade (COSTA G, et al., 2018).

Esses números superiores encontrados no sexo masculino estão, por exemplo, a Atenção Primária à Saúde (APS), os homens costumam protelar a procura de atendimentos a esses serviços, o que leva ao aumento da demanda nos serviços de emergência, tanto por doenças agudas, como pela agudização de doenças já instaladas (CAVALCANTE AKCB, et al., 2015; DUARTE SJH, et al., 2011).

Ao levar em consideração a associação do sexo com o diagnóstico, este estudo corrobora com outros autores, que mostram que o trauma acomete quatro vezes mais homens do que mulheres; além disso, quando associados a faixa etária e o diagnóstico, observa-se que as emergências traumáticas são maiores na população jovem, com predominância nessa pesquisa para a faixa etária entre 18 e 59 anos, enquanto as clínicas são diretamente proporcionais ao aumento da idade (DIAS JMC, et al., 2016; GUIZZO WA, et al., 2020).

No que se refere à faixa etária, existe uma predominância nos atendimentos à população acima dos 60 anos, o que corrobora com a pirâmide etária do país, que manteve a tendência de envelhecimento. Com o avanço da idade, existe maior proporção de acometimentos por comorbidades na população. Esta afirmação é expressada com os dados desta pesquisa, que evidenciam que as pessoas, em sua grande maioria, são portadoras de mais de uma comorbidade, sendo as principais a HAS e a DM (MELO LA, et al., 2017; NUNES BP, et al., 2016).

Considerando as adversidades relacionadas ao envelhecimento, a HAS e a DM se destacam como causas importantes de morbimortalidade e complicações cardiovasculares, juntamente com fatores de riscos como tabagismo, dislipidemia e obesidade (FERREIRA JDF, et al., 2017).

Segundo a Organização Mundial da Saúde (OMS), a HAS afeta de $20 \%$ a $40 \%$ da população adulta, e neste estudo o número se sobressaiu em 14\%; infere-se que isso ocorra pelo fato de que não estão sendo considerada a população toda, e sim apenas aqueles que tiveram algum acometimento, além disso, as maiores prevalências estão entre os homens e em países de média e baixa renda. Esses números justificam a crescente demanda por serviços de emergência nos últimos anos (FIORIO CE, et al., 2020).

Quanto à natureza dos atendimentos, destaca-se a predominância de atendimentos clínicos. Esse resultado é semelhante ao da pesquisa realizada no norte de Minas Gerais, onde 56,7\% dos atendimentos foram clínicos. E no estudo realizado em um município da Região Metropolitana de Curitiba, que teve 61,6\% de atendimentos clínicos (TIBÃES HBB, et al., 2018; SILVA JS, et al., 2020).

Uma particularidade de Curitiba é que o município conta com o Serviço Integrado ao Trauma em Emergência (SIATE), serviço vinculado ao Corpo de Bombeiros do Paraná, criado em 1990, que foi o pioneiro nesse tipo de atendimento e serviu de exemplo nacionalmente, voltado exclusivamente para o atendimento de trauma. O SIATE possui Central de Regulação própria, o atendimento a esse serviço é solicitado via ligação telefônica ao número 193, atua juntamente ao SAMU e, apesar de ambos atenderem no município, suas funções são distintas, porém complementares. (GUIZZO WA, et al., 2020).

Quanto aos diagnósticos, destacam-se a Dor Torácica e o Acidente Vascular Cerebral (AVC). Ambas estão dentro da classificação de Doenças Cardiovasculares (DCVs). No Brasil as DCV são a principal causa de morte desde o final da década de 60 , essa estatística está relacionada com as mudanças no estilo de vida e uma maior expectativa de vida da população (RIBEIRO ALP, et al, 2015).

A Dor Torácica é um dos principais sintomas do Infarto Agudo do Miocárdio (IAM). As mortes por essa patologia ocorrem nas primeiras horas de surgimento da doença, sendo 40 a $65 \%$ na primeira hora e aproximadamente $80 \%$ nas primeiras 24 horas. Deste modo, o período desde o início dos sintomas até a definição do tratamento é diretamente proporcional ao prognóstico do paciente (PIEGAS LS, et al., 2015). 
O Eletrocardiograma (ECG) é um exame rápido e fácil de ser realizado no atendimento pré-hospitalar. Esse, quando realizado e avaliado por um médico habilitado ainda fora do âmbito intra-hospitalar, reduz em $34 \%$ o tempo porta-agulha (tratamento fibrinolítico) e em $18 \%$ o tempo porta-balão (angioplastia). Portanto, é essencial que os atendimentos aos pacientes com suspeita desse diagnóstico sejam atendidos pelas USAs, pois esse serviço conta com equipe e materiais adequados para dar suporte a esses pacientes (PIEGAS LS, et al., 2015).

No atendimento ao AVC também não é diferente, os pacientes com suspeita desse diagnóstico precisam de atendimento médico de forma rápida. A terapia indicada em casos de AVC isquêmico é a trombólise, contudo, esse tratamento precisa ser iniciado em até 4,5 horas do início dos sintomas. Nesse caso, o APH precisa ser rápido na identificação do diagnóstico e transporte até o serviço de referência (SARMENTO SDG, et al., 2017).

Para garantir o atendimento adequado aos pacientes levando em conta seus critérios de gravidade, 0 SAMU Regional Metropolitano-PR tem estabelecido desde 2017, procedimentos operacionais padrões (POP) para os casos de IAM, AVC, Hemorragia Digestiva, dentre outros. Estes protocolos visam ao atendimento apropriado e encaminhamento até o serviço de referência, independente do ponto da rede de urgência e emergência em que o paciente procurou o primeiro atendimento (CURITIBA, 2017).

Nesta pesquisa $47 \%(n=184)$ dos atendimentos foram ocorrências (atendimento primário), atendimentos realizados fora do âmbito hospitalar, como em domicílio e vias públicas. A Resolução do CFM 2110/14 determina que os atendimentos nesses locais, por ordem de complexidade, devem ser priorizados em relação às transferências (atendimento secundário) de pacientes na rede, sendo essa decisão uma atribuição do médico regulador (CONSELHO FEDERAL DE MEDICINA, 2014).

As transferências de pacientes foram de $(n=203 ; 52,5 \%)$. A transferência é entendida como o transporte de pacientes entre unidades não hospitalares ou hospitalares de atendimento às urgências e emergências. Pacientes com casos de gravidade sempre deverão ser transportados pelas USAs devido à sua complexidade e necessidades (BRASIL, 2002).

As Unidades de Pronto Atendimento (UPAs) compõem a rede de atenção à saúde, sendo o componente pré-hospitalar fixo, ocupam o nível intermediário da estrutura hierárquica do sistema de saúde, prestando assistência de média complexidade. Funcionam como ponto de estabilização e acolhimento dos pacientes que necessitam de atendimento de emergência na rede. Quando o atendimento de alta complexidade faz-se necessário, através de fluxos e protocolos específicos, encaminham por meio do SAMU os pacientes para serviços de maior complexidade (OLIVEIRA SN, et al., 2015).

A demanda de transferências ocupa uma grande porcentagem dos atendimentos realizados pelo SAMU, deste modo é necessária uma adequada comunicação entre os serviços da rede bem como a boa organização na referência e contra referência dos pacientes, com a finalidade de evitar a sobrecarga do serviço (CASTRO $R R$, et at., 2020).

O óbito também está entre os diagnósticos mais prevalentes, sendo $8,2 \%(n=30)$. Esse alto número acontece porque é função do médico intervencionista do SAMU a verificação e constatação do óbito em Curitiba, também cabe ao médico o preenchimento da Declaração de Óbito (DO) de pacientes que foram a óbito por causa natural, em casos onde o paciente não possui acompanhamento médico e nos momentos em que a unidade de saúde de referência esteja fechada. Essa informação justifica o alto índice de diagnósticos de óbitos na pesquisa (CURITIBA, 2014).

O Município de São José dos Pinhais, que faz parte da região metropolitana de Curitiba, conta desde 2005 com o Serviços de Verificação de Óbito e Esclarecimento da Causa Mortis (SVO). O SVO tem como função determinar a causa e realizar o preenchimento da declaração de óbito, em casos de morte natural, com ou sem assistência médica, que não possuem sinais de violência. Em vista disso, esse serviço diminui a sobrecarga das USAs, provendo um maior tempo de disponibilidade para atendimentos que ofereçam risco de vida imediato. É importante destacar que os demais municípios possuem fluxos específicos (NUNES BP, et al., 2016). 
A ficha de atendimento utilizada pelo serviço faz parte do prontuário do paciente, sendo a única forma de registro no Atendimento Pré-Hospitalar (APH). A função deste documento é deixar registradas todas as informações a respeito do atendimento fornecido. É considerado um documento legal e por essa razão necessita que as informações sejam preenchidas de forma clara e completa (BARROS BC, et al., 2014).

Neste estudo a limitação foi relacionada ao preenchimento inadequado das fichas, pois muitos campos não foram preenchidos pelos profissionais, dificultando a tabulação/análise de diversas variáveis que poderiam contribuir com a pesquisa. Também havia fichas com letra ilegível e informações em campos trocados. No entanto, destaca-se que não foram encontradas fichas danificadas durante a coleta de dados, sendo este um ponto positivo.

Faz-se necessário, portanto, desenvolver mecanismos para estabelecer o preenchimento correto e completo da ficha, seja por atividades de capacitação contínua demonstrando a importância de tais informações, bem como desenvolver um modo mais rápido e fácil de preenchimento. A utilização de aparelhos tecnológicos, como tablet ou celular, com formulários de preenchimento por checklist de campos obrigatórios pode ser uma opção.

\section{CONCLUSÃO}

Conclui-se, neste trabalho, que as USAs do SAMU Regional Metropolitano/PR realizaram predominantemente atendimentos clínicos, e esses foram diretamente proporcionais ao aumento da idade. Dor torácica, Acidente Vascular Cerebral e Óbito foram os diagnósticos mais atendidos pelas equipes. Quanto ao objetivo do chamado, a maioria foi de transferências (atendimento secundário). Levando em consideração os principais diagnósticos, é notável que as USAs cumprem seu papel de assistência ao paciente de maior gravidade. Aponta-se a necessidade de aperfeiçoamento no preenchimento da ficha, considerando que ela é o prontuário do paciente no APH e as informações do atendimento realizado são de grande importância na continuidade da assistência.

\section{REFERÊNCIAS}

1. ABREU MSS, et al. Perfil das vítimas de acidente motociclístico atendidas pelo serviço de atendimento móvel de urgência. Revista Eletrônica Acervo Saúde, 2019; (37): e1560.

2. BATTISTI GR, et al. Perfil de atendimento e satisfação dos usuários do Serviço de Atendimento Móvel de Urgência (SAMU). Rev Gaúcha Enferm. 2019; 40: e20180431.

3. BARROS BC, et al. O instrumento de registro do Samu: com a palavra dos profissionais de saúde. J res: fundam. care online, 2014; 618-626, 2014; 6(2): 618-626.

4. BRASIL. Portaria n. 2.048, de 02 de novembro de 2002. Dispõe sobre o Regulamento Técnico dos Sistemas Estaduais de Urgência. Brasília: Ministério da Saúde; 2002. Disponível em: https://bvsms.saude.gov.br/bvs/saudelegis/gm/2002/prt2048_05_11_2002.html. Acesso em: 26 jul. 2021

5. BRASIL. Portaria n. 1.864, de 29 de setembro de 2003. Institui o componente pré-hospitalar móvel da PNAU SAMU Brasília: Ministério da $\quad$ Saúde; $2003 . \quad$ Disponível https://bvsms.saude.gov.br/bvs/saudelegis/gm/2003/prt1864_29_09_2003.html. Acesso em: 25 jul 2021

6. BRASIL. Portaria n. 354, de 10 de março de 2014. Pública a proposta de Projeto de Resolução "Boas Práticas para Organização e Funcionamento de Serviços de Urgência e Emergência". Disponível em: https://bvsms.saude.gov.br/bvs/saudelegis/gm/2014/prt0354_10_03_2014.html. Acesso em: 22 jul de 2021 ,

7. BRASIL. Portaria n. 1.405, de 29 de junho de 2006. Institui a Re⿳亠 Nacional de Serviços de Verificação de Óbito e Esclarecimento da Causa Mortis (SVO). Brasília: Ministério da Saúde; 2006. Disponível em: https://bvsms.saude.gov.br/bvs/saudelegis/gm/2006/prt1405_29_06_2006.html. Acesso em 26 de jul. 2021.

8. BRASIL. Resolução 466, de 12 de dezembro de 2012. Aprovar as seguintes diretrizes e normas regulamentadoras de pesquisas envolvendo seres humanos. Brasília: Ministério da Saúde; 2012. Disponível em: https://bvsms.saude.gov.br/bvs/saudelegis/cns/2013/res0466_12_12_2012.html. Acesso em: 25 jul. 2021

9. CAVALCANTE AKCB, et al. Perfil dos acidentes de trânsito atendidos pelo pré-hospitalar móvel. Revista Baiana de Enfermagem, 2015; 29(2): 135-145.

10. CONSELHO FEDERAL DE MEDICINA. Resolução CFM 2.110 de 19 de novembro de 2014. Normatização do funcionamento dos Serviços Pré-Hospitalares Móveis de Urgência e Emergência, em todo o território nacional. Brasília, Conselho Federal de Medicina; 2014.2 Disponível em: https://sistemas.cfm.org.br/normas/visualizar/resolucoes/BR/2014/2110. Acesso em: 24 de jul. 2021

11. COSTA G, et al. Mobile emergency care service: A time-course assessment and characterization of demand. International Emergency Nursing, 2018; 41: 45-50. 
12. CASTRO, et al. Caracterização das ocorrências do Serviço de Atendimento Móvel de Urgência - SAMU. Revista Eletrônica Acervo Enfermagem, 2020; 7, e5625.

13. CURITIBA. Serviço de Urgência e Emergência de Curitiba. Manual de Procedimentos Operacional Padrão - Fluxo de Regulação, 2017.

14. CURITIBA. Instrução normativa N.3 de 1 de setembro de 2014. Regulamenta fluxos e responsabilidades na confirmação do óbito e preenchimento da declaração de óbito - no âmbito da Secretaria Municipal de Saúde e nos estabelecimentos de saúde que prestam atendimento aos usuários do SUS Curitiba. Diário Oficial, 2014; n.166 - ANO III. Disponível em: https://legisladocexterno.curitiba.pr.gov.br/DiarioConsultaExterna_Pesquisa.aspx. Acesso em: 25 jul. 2021

15. DIAS JMC, et al. Profile of state prehospital mobile emergency care service. Cogitare Enfermagem, 2016; 21(1): 1-8.

16. DORR MR, et al. Serviço de atendimento móvel de urgência do Rio Grande do Sul. Revista Enfermagem em foco, $2020 ; 11(2): 76-82$.

17. FERREIRA JDF, et al. Fatores de risco para doenças cardiovasculares em idosos. J Nurs UFPE on line, 2017; 11(12): 4895-905.

18. FERREIRA AM, et al. Serviço de atendimento móvel de urgência: satisfação de usuários. Rev Enferm UFPE online. 2017; 11(10):3718-3724.

19. FIORIO CE, et al. Prevalência de hipertensão arterial em adultos no município de São Paulo e fatores associados. Rev bras. epidemiol, 2020; 23: e200052.

20. GUIZZO WA, et al. Trauma em Curitiba: avaliação multifatorial de vítimas admitidas em um hospital universitário. Rev. Col Bras Cir, 2020; 47: e20202408.

21. MELO LA, et al. Fatores socioeconômicos, demográficos e regionais associados ao envelhecimento populacional. Rev. bras. geriatr. gerontol., 2017; 20(4): 493-501.

22. NUNES BP, et al. Multimorbidity and mortality in older adults: a systematic review and meta-analysis. Arch Gerontol Geriatr, 2016; 67: 130-138.

23. OLIVEIRA SN, et al. Unidade de Pronto Atendimento - UPA 24h: percepção da enfermagem. Texto contexto enfermagem, 2015; 24(1).

24. PIEGAS LS, et al. V Diretriz da Sociedade Brasileira de Cardiologia sobre o tratamento do infarto agudo do miocárdio com supra desnível do segmento ST. Arq Bras Card, 2015; 105(2), Supl. 1.

25. R CORE TEAM. R: A Language and Environment for Statistical Computing. (2020). Disponível em: http://www.rproject.org/index.html. Acesso em: 21 fev. 2021.

26. REZENDE DA, et al. Gestão integrada do transporte público coletivo e relações com cidade digital estratégica: Curitiba e região metropolitana. Redes St. Cruz do Sul Online, 2021. v. 26.

27. RIBEIRO ALP, et al. Cardiovascular Health in Brazil: Trends and Perspectives. Circulation, 2016; 133(4): $422-433$.

28. SARMENTO SDG, et al. Profile of individuals with neurological disorders assisted by a prehospital mobile emergency care service. Cogitare Enfermagem, 2017; 22(2): e49698.

29. DUARTE SJH, et al. Atendimentos prestados pelo serviço móvel de urgência em Cuiabá, MT, Brasil. Revista Eletrônica de Enfermagem, 2011; 13(3): 502-7.

30. SILVA JS, et al. Perfil dos atendimentos realizados pelo Serviço de Atendimento Móvel de Urgência no município de Colombo (PR). Revista Espaço para a Saúde, 2020; 21(1):26-33.

31. SOARES MKP, et al. Perfil dos usuários atendidos por um serviço pré-hospitalar móvel de urgência no nordeste brasileiro. J res: fundam. Care online, 2018; 10(2): 503-509.

32. TIBÃES HBB, et al. Service Profile of the Mobile Emergency Care Service in The North of Minas Gerais State. J res: fundam. care online, 2018; 10(3): 675-682. 